\title{
La qualité de vie pour tous
}

\section{Raymond Leblanc}

Le paradoxe des humains est que l'on essaie de parler de l'humain en général alors que, dans le même temps, tout être humain est unique, un individu. Au-delà de l'ultra-individualité de l'expérience, comment peut-on donner à celle-ci une sorte de signification universelle et transcendantale? Notre vision de l'humain est utopique. En effet, nous croyons au courage et à la solidarité foncière de l'humain.

Jack Riemer, un théologien juif, a su exprimer cette audace de l'humain dans ce beau poème, qui s'intitule Dieu, nous ne pouvons pas vraiment te prier (2002) :

Ô Dieu, nous ne pouvons pas vraiment te prier pour que cesse la guerre, car nous savons que tu as fait le monde de telle façon que l'homme doive trouver son chemin de la paix, tant en lui-même qu'avec son voisin.

Ô Dieu, nous ne pouvons pas vraiment te prier pour que cesse la famine, car tu nous as donné assez de ressources pour nourrir le monde entier, si seulement nous les utilisions avec sagesse.

Ô Dieu, nous ne pouvons pas vraiment te prier d'éradiquer l'injustice, car tu nous as donné des yeux capables de voir le bien dans chaque créature, si seulement nous les utilisions avec sagesse.

Nous ne pouvons pas vraiment te prier, ô Dieu, de faire cesser le désespoir, car tu nous as donné le pouvoir de transformer les taudis et de semer l'espérance, si seulement nous les utilisions avec sagesse.

Nous ne pouvons pas vraiment te prier, ô Dieu, de faire cesser les maladies, car tu nous as donné une intelligence capable d'imaginer et de créer des médicaments, si seulement nous les utilisions avec sagesse. 
C'est pourquoi, ô Dieu, nous te prions plutôt de nous donner force, détermination et courage d'agir et de ne pas simplement prier, d'être plutôt que de simplement espérer.

Le paradoxe de l'humain, comme celui de la vie, c'est son incommensurabilité et son indivisibilité. Le concept de qualité de vie semble simple... Il pourrait se traduire comme suit : Je suis satisfait de ma vie et de ce que la vie m'offre comme projet ou comme avenir. Pourtant, aucune définition consensuelle ni aucune mesure commune de la qualité de vie ne recueillent l'assentiment de tous. Même si un survol historique de la conception de la qualité de vie au $\mathrm{XX}^{\mathrm{e}}$ siècle associe celle-ci à l'amélioration des conditions de vie (jusqu'en 1950), à la valorisation des rôles sociaux (1970-1990) et, aujourd'hui, à la participation citoyenne, et ce, dans une narration de mondialisation capitaliste où l'humain est une ressource, le droit au travail ne peut plus être garanti à tous et la sécurité sociale en est amputée. Selon Petrella (2007), la citoyenneté n'admettrait pas de hiérarchie ni de classement, et il serait impensable de construire une société avec des citoyens de $1^{\mathrm{er}}$, de $2^{\mathrm{e}}$ ou de $3^{\mathrm{e}}$ ordre.

Dans notre monde ultralibéral, le travail sort du champ social. Tout est marchandé. L'éducation est devenue un bien économique. Fort de ce constat, la qualité de vie devrait devenir un levier visionnaire pour améliorer les conditions de vie de tous, et en particulier celles des plus vulnérables de notre planète. En 2000, Goode, Magerotte et nous-même avons publié un livre intitulé Qualité de vie pour les personnes présentant un handicap : perspectives internationales, dans lequel étaient rassemblés les résultats d'études menées dans dix pays différents, dont le Canada, et portant sur la façon dont la qualité de vie est utilisée dans les services et dans la recherche. Les contributions abordent aussi l'usage de la qualité de vie dans différents domaines, tels que l'autodéfense, le handicap mental sévère et profond, les problèmes de comportement, l'intervention précoce, l'école, les personnes adultes et âgées, la formation du personnel, l'évaluation, la recherche ainsi que les applications du concept.

Au niveau mondial, le rapport intitulé Factor Four-Doubling Wealth, Halving Resource Use (Weiszacker, Lovins et Lovins, 1997) publié par un centre de recherche allemand réputé (Wüppertal Institut für Unwelt), mentionne le fait que la qualité de vie de nos sociétés doublerait si nous diminuions de moitié la quantité de ressources utilisées pour 
produire l'actuel niveau de développement. Petrella (2007) nous exhorte de rejeter le rêve américain qui

produit $26 \%$ des émissions mondiales de $\mathrm{CO} 2$ pour une population n'atteignant que $5 \%$ de la population mondiale; la consommation de 801 litres d'eau potable par jour par habitant (alors que la consommation des Suédois se situe à 119 litres, celles des Belges à 106 litres et celle des Allemands à 130 litres par jour par personne); la production de $932 \mathrm{~kg}$ de déchets ménagers par an par personne (alors que la quantité de ces déchets produits par les Italiens, fort producteurs eux aussi de ces déchets, s'élève à $350 \mathrm{~kg}$ par an par personne); une dépense annuelle de 500 milliards de dollars en armement. » (p. 102)

Selon Bauman (2004), la population relativement modeste des pays les plus affluents brûlerait deux tiers de la totalité de l'énergie. La qualité de vie devient alors un impératif moral. Pour une nouvelle narration d'un monde meilleur, les six principes suivants devraient être prioritaires : le principe de la vie, le principe de l'humanité, le principe du vivre ensemble, le principe des biens communs, le principe de la démocratie et le principe de la responsabilité. Il faut reconnaître les droits inhérents à la personne humaine et aux communautés humaines qui forment les droits de citoyenneté. Versant matériel, ces droits sont l'eau, l'air, la nourriture, le logement. Versant immatériel, ce sont les droits à l'éducation, au travail, à la liberté de pensée et de parole, à la santé et à la liberté de mouvement. Relatif au vivre-ensemble, nous devons promouvoir le droit à la paix, le droit à toutes les formes de sécurité, qu'elles soient économique et culturelle, ainsi que le droit aux libertés.

L'insertion à la citoyenneté dans le développement des enfants et des adolescents est entravée par des conditions de vie liées à la pauvreté, comme la sous-stimulation, la négligence, la violence, le fait de vivre dans des environnements moins sécuritaires et de fréquenter des institutions moins compétentes. Par ailleurs, des études longitudinales ont démontré qu'un environnement où règnent la dépression, la détérioration subséquente des relations parentales et le manque de soutien mènent à une dégradation de la qualité des conduites des parents envers leurs enfants.

Et si l'on poussait plus loin la réflexion, comme l'a fait le psychologue Csikszentmihalyi (1990) par rapport à son concept d'expérience optimale, la quête d'expériences de travail et de loisirs qui mobilisent nos capacités nous apporte un défi. Ainsi, le flow, également appelé l'expérience de bien-être intense, est suscité par des activités créatives où la 
personne fait face à un défi qui l'interpelle et pour lequel elle est convaincue de posséder les compétences nécessaires pour le relever. La question à se poser est alors la suivante : «Comment chacun peut-il se créer une vie de qualité? » Nous concevons la qualité de vie de la même façon que l'exprime notre amie Marie-Claire Haelewyck, professeure à l'Université de Mons-Hainaut, en Belgique : c'est «un voyage lié étroitement à notre condition humaine».

La qualité de vie est un construit multidimensionnel composé de deux niveaux généraux : au niveau d'une population et au niveau de l'individu. Comme construit au niveau d'une population (mondial et national), deux ordres d'indicateurs ont cours. Une conception d'origine scandinave cible des indicateurs sociaux et objectifs, tels que l'argent disponible, la propriété, la sécurité et la densité du réseau social de la personne. Une seconde conception, venant des États-Unis, est centrée sur la personne et vise à relever l'évaluation du degré de satisfaction et de bien-être de celui-ci, comme la liberté de choisir, le réseau d'amis, sa contribution à la communauté, le contrôle sur son milieu de vie, le contrôle de ses activités, ses besoins émotionnels ainsi que le fait d'être accepté par les membres de sa famille, ses employeurs et la communauté locale. Les indicateurs subjectifs et psychologiques sont déterminants dans le cadre de cette seconde conception. L'économiste Boulding (1981) propose que la qualité de vie en lien au revenu par personne soit le critère majeur du progrès économique. Toutefois, nous savons que la qualité de vie va au-delà du confort matériel, de la santé et de la longévité. Salvaris (2000) propose un cadre global d'indicateurs de bien-être composé de dix dimensions : 1) santé individuelle et bien-être; 2) possibilités d'emploi; 3) société équitable; 4) société sécuritaire; 5) économie responsable; 6) bon gouvernement et mise en vigueur de lois; 7) environnement durable; 8) communautés en santé et citoyens actifs; 9) vie culturelle vigoureuse; 10) bonnes relations internationales.

Comme construit individuel, la qualité de vie se traduit par le fait de se sentir bien et d'avoir à sa disposition ce qui est nécessaire pour vivre sa vie, et ce, de la meilleure façon possible. Au niveau individuel, la qualité de vie se définit localement et se traduit par le fait de se sentir bien et de vivre sa vie de la meilleure façon possible.

La qualité de vie comprend une dimension psychologique interne, à savoir l'état psychique de la personne, sa dignité, ses valeurs et ses préférences. Il faudrait ajouter que 
certains indicateurs sont interactifs, par exemple le soutien social et l'accord entre la personne et les exigences de son milieu. Il faut donc adopter une perspective holistique de la qualité de vie.

Zapf (1977) propose d'abord que la dimension du bien-être objectif reflète les conditions de vie, soit bonnes ou mauvaises, que vit une personne; ensuite, que la dimension subjective soit jugée positive ou négative par la personne, c'est-à-dire positive lorsqu'elle est associée à se sentir bien adaptée et négative lorsque la personne ressent de la dissonance et qu'elle subit des privations dans sa vie. Ainsi, quatre conditions de vie générales, deux positives et deux négatives, peuvent se manifester. Les conditions positives sont d'abord le bien-être lorsque la personne jouit de bonnes conditions de vie objectives et exprime son état positif par un «j' aime ma vie » et, ensuite, la personne adaptée qui vit de mauvaises conditions de vie mais se dit quand même heureuse, phénomène souvent considéré comme le paradoxe de la satisfaction ou le paradoxe du handicap. À ce sujet, notons les deux exemples suivants : d'abord, les personnes qui vivent avec des handicaps divers manifestent habituellement un niveau de bien-être satisfaisant; ensuite, après une période de choc, les victimes d'accidents variés retrouvent le même niveau général de bien-être qu'ils avaient avant l'accident (Myers et Diener, 1997). Ainsi, nous pouvons constater que chaque personne exerce un contrôle sur sa vie. Les conditions négatives sont la dissonance chez la personne qui jouit de bonnes conditions de vie, mais qui se dit malheureuse (très souvent, la personne s'ennuie, est apathique, est inquiète ou, encore, est anxieuse) et la privation chez celui qui vit de pauvres conditions de vie et qui se dit totalement insatisfait de sa vie. En somme, le bonheur ou le bien-être se définit par « la présence d'affects positifs et l'absence relative d'affects négatifs ainsi que par un sens global de satisfaction de vivre » (Myers et Diener, p. 41).

Dans une perspective de développement, l'enfant trouve son bonheur dans le jeu, l'adolescent dans ses appartenances ainsi que ses amitiés et l'adulte dans le travail. Il ne faut pas oublier les personnes âgées ainsi que celles qui font partie de la nouvelle communauté constituée de personnes âgées de plus de 85 ans. Pour tous, et tout particulièrement pour les enfants, le contact fréquent avec la nature et avec le naturel (par exemple, les animaux) génère un respect envers la nature, une valeur essentielle à la vie. Somerville (2006, p. 113) ajoute que « being connected with nature can teach us to live 
more comfortably with uncertainty », qu'être connecté avec la nature peut nous enseigner à vivre plus confortablement malgré l'incertitude (traduction libre). Pour ce qui est des personnes plus âgées, la satisfaction reliée à jouir de bonnes relations sociales et à vivre en santé importent sans doute grandement. Il faut aussi envisager que ces temps de vie apportent des bénéfices cumulatifs. Chez les plus âgés, le jeu, les amis, le travail et la santé sont porteurs de bonheur; chez le jeune enfant, seul le système de jeu encadre son univers. Ainsi les expériences de bonheur sont-elles des apprentissages, qui ne sont pas des cadeaux du ciel. L'humain doit apprendre à améliorer ses contacts et ses réseaux avec ses proches. Il faut apprendre à développer des amitiés fortes et un réseau social étendu. En effet, un humain vit de bonnes conditions de vie lorsqu'il jouit et régule un réseau social riche en fréquence et en variété et que ses rapports entraînent une découverte positive de soi, de son importance aux yeux des autres.

Il faut aussi apprendre à collaborer. Il faut cultiver des plaisirs avec les saveurs et les fleurs. Hughes, Hwang, Kim, Eisenman et Killiam (1995) ont rapporté quinze dimensions qui marquent la qualité de vie renforçant ainsi un grand nombre d'indicateurs. Nous les présentons par ordre décroissant : le bien-être psychologique et la satisfaction personnelle; les interactions sociales; 1'emploi; le bien-être physique et matériel; l'autodétermination et les choix personnels; le sentiment de compétence personnelle; l'ajustement communautaire et l'indépendance; l'intégration communautaire; l'acceptation sociale; le statut et l'adaptation écologique; le développement et la satisfaction personnelle; l'environnement résidentiel; les loisirs; la normalisation; les indicateurs individuels et démographiques; la responsabilité civile; les services de soutien reçus.

Sur le travail ou le métier, le beau passage suivant de Kundera (1998) figurant dans le roman intitulé $L$ 'Identité mérite réflexion :

Parce que les métiers de jadis, au moins pour une grande part, n'étaient pas pensable sans un attachement passionnel : les paysans amoureux de leur terre; mon grand-père, le magicien des belles tables; les cordonniers qui connaissaient par cœur les pieds de tous les villageois; les forestiers; les jardiniers; je suppose mêmes les soldats tuaient alors avec passion. Le sens de la vie n'était pas une question, il était avec eux naturellement, dans leurs ateliers, dans leurs champs. Chaque métier avait cédé sa propre mentalité, sa propre façon d'être. Un médecin pensait autrement qu'un paysan, un militaire avait un autre comportement qu'un instituteur. Aujourd' hui nous sommes tous pareils, tous unis par la commune indifférence envers notre travail. (1998, p. 104) 
Heureux celui qui aime son travail, car le travail occupe environ un tiers de notre temps de vie.

Milan Kundera, Patrick Modiano, Jean-Marie Gustave LeClézio et John Irving sont les écrivains que nous lisons et relisons depuis longtemps, et chaque relecture nous apporte une nouvelle expérience. Quindlen (1998) exprime avec acuité l'apport de ces auteurs à notre vie :

In books I have travelled, not only to other worlds, but into my own. I learned who I was and who I wanted to, what I might aspire to, and what I might dare to dream about my world and myself. But I felt that I, too, existed much of the time in a different dimension from everyone else I knew. There was waking, and there was sleeping. And then there were books, a kind of parallel universe in which anything might happen and frequently did, a universe in which I might be a newcomer but was never really a stranger. My real, true world. My perfect island. (p. 61)

Modiano à la recherche inlassable de ses racines, LeClezio en communion panthéiste avec la nature et Irving, un chevalier en quête du Graal, sèment l'insatiable aventure de nos imaginaires. Nous sommes dans une large mesure ce que nous lisons. Milan Kundera (2002) dans son roman intitulé L'Ignorance nous rappelle ce qui suit :

If someone could retain in his memory everything he had experienced, if he could any time call up any fragment of his past, he would be nothing like human beings; neither his loves nor his friendships nor his angers nor his capacity to forgive or avenge would resemble ours. (p. 122-123)

Nous souscrivons à la philosophie de vie d'une éthique partagée, ou communément appelée de valeurs communes, misant tout spécialement sur l'espoir proposé par Somerville (2006). Cette éthique partagée est fondée sur deux principes et deux concepts complémentaires : d'une part, le profond respect pour toute forme de vie et un respect pour l'esprit humain; d'autre part, la reconnaissance et la croissance d'un sens du sacré et de l'ordre du naturel. L'histoire suivante, racontée par Somerville, est un appel à une éducation des jeunes à l'ordre du naturel :

Une jeune fille de onze ans a écrit dans le journal de classe n'avoir jamais grimpé dans un arbre ni vu un coucher de soleil. Elle en rêvait. Son enseignante a découvert 
qu'un grand nombre de ses élèves étaient dans la même situation, certains n'avaient jamais même jamais marché dans un champ. Ils ne sentaient aucune affinité avec le monde naturel, sa fragilité ni sa valeur inestimable. En fait, ils avaient peur de la nature. Je crains qu'un grand nombre d'enfants n'éprouvent pas pour la nature cet attachement qui engendre le respect de l'ordre naturel nécessaire à la préservation de certaines de nos valeurs partagées les plus importantes... les jeunes sont avides d'éprouver la crainte et la fascination de la nature et nous pouvons les aider à y parvenir. C'est plus qu'une promesse, nous en avons l'obligation morale. (p. 91)

Cette découverte apporte un bonheur de vivre.

\section{Références}

Bauman, Z. (2004). Wasted Lives (Modernity and its Outcasts). Cambridge, England: Polity Press.

Boulding, K. (1981). Human Knowledge as a Special System. Systems Research and Behavioral Science, 26(2), 93-102.

Csikszentmihalyi, M. (1990). Flow: The Psychology of Optimal Experience. New York, NY: Harper Collins.

Goode, D., Magerotte, G. et Leblanc, R. (2000). Qualité de vie pour les personnes présentant un handicap: perspectives Internationales. Bruxelles, Belgique : De Boeck Supérieur.

Haelewyck, M. HC. Atelier sur la qualité des services. Revue francophone de la déficience intellectuelle, juin, 91-94.

Hughes, C., Hwang, B., Kim, J., Eisenman, L.T., et Killiam, D.J. (1995). Quality of life in applied research: A review and analyses of empirical measures. American Journal of Mental Retardation, 99(6), 623-641.

Kundera, M. (1998). L’Identité. Paris, France : Gallimard.

Kundera, M. (2003). L'Ignorance. Paris, France : Gallimard. 
Myers, D.G. et Diener, E. (1997). The pursuit of happiness. New research uncovers some anti-intuitive insights into how many people are happy and why. Scientific America, p. $38-43$.

Petrella, R. (2007). Pour une nouvelle narration du monde. Montréal, Québec : Écocité.

Quindlen, A. (1998). How Reading Changed My life. New York: New York, Ballantine.

Riemer, J. (2002). Jewish Insights on Death and Mourning. New York, NY: Schocken Books.

Salvaris,R. (2000). Community and Social Indicators. How citizens can measure progress. An overview of social and community indicator projects in Australia and internationally. Institute for Social research. Swinburne University of technology.

Somerville, M. (2006). The Ethical Imagination (Journeys of the Human Spirit). Toronto, Ontario: House of Anansi Press.

Weiszacker, E. van., Lovins, A.B. et Lovings L. H. (1997). Factor Four-Doubling Wealth Halving Resource Use. A New Report to the Club of Rome. London, England: Earthscan.

Zapf, D. (1977). Soziale Indikatoren-eine Zwischenbilanz. In : H. J. Krupp \& W Zapf (eds.) Soziapolitik und Sozialrichtertatung. Frankfurt am Main and New York : Campus, p. 231-246. 